Audiology

Neurotology
Audiol Neurotol 2020;25:96-108

DOI: $10.1159 / 000503846$
Received: August 19, 2019

Accepted after revision: October 1, 2019

Published online: January 22, 2020

\title{
Interactions between Auditory and Vestibular Modalities during Stimulation with a Combined Vestibular and Cochlear Prosthesis
}

\author{
James O. Phillips ${ }^{a, c}$ Leo Ling ${ }^{a}$ Amy Nowack ${ }^{a}$ Brenda Rebollarc \\ Jay T. Rubinstein ${ }^{a, b}$ \\ ${ }^{a}$ Department of Otolaryngology, University of Washington, Seattle, WA, USA; ${ }^{b}$ Department of \\ Bioengineering, University of Washington, Seattle, WA, USA; ${ }^{C}$ Center for Integrative Brain Research, \\ Seattle Children's Hospital, Seattle, WA, USA
}

\section{Keywords}

Vestibular prosthesis - Cochlear prosthesis - Implant . Human

\begin{abstract}
Background: A combined vestibular and cochlear prosthesis may restore hearing and balance to patients who have lost both. To do so, the device should activate each sensory system independently. Objectives: In this study, we quantify auditory and vestibular interactions during interleaved stimulation with a combined 16-channel cochlear and 6-channel vestibular prosthesis in human subjects with both hearing and vestibular loss. Methods: Three human subjects were implanted with a combined vestibular and cochlear implant. All subjects had severe-to-profound deafness in the implanted ear. We provided combined stimulation of the cochlear and vestibular arrays and looked for interactions between these separate inputs. Our main outcome measures were electrically evoked slow-phase eye velocities during nystagmus elicited by brief trains of biphasic pulse stimulation of the vestibular end organs with and without concurrent stimulation of the cochlea, and Likert scale assessments of perceived loudness and pitch during stimulation of the cochlea,
\end{abstract}

with and without concurrent stimulation of the vestibular ampullae. Results: All subjects had no auditory sensation resulting from semicircular canal stimulation alone, and no sensation of motion or slow-phase eye movement resulting from cochlear stimulation alone. However, interleaved cochlear stimulation did produce changes in the slow-phase eye velocities elicited by electrical stimulation. Similarly, interleaved semicircular canal stimulation did elicit changes in the perceived pitch and loudness resulting from stimulation at multiple sites in the cochlea. Conclusions: There are significant interactions between different sensory modalities during stimulation with a combined vestibular and cochlear prosthesis. Such interactions present potential challenges for stimulation strategies to simultaneously restore auditory and vestibular function with such an implant.

$$
\begin{aligned}
& \text { () } 2020 \text { The Author(s) } \\
& \text { Published by S. Karger AG, Basel }
\end{aligned}
$$

\section{Introduction}

Auditory and vestibular loss often occur together. The end organs of the auditory and vestibular systems within the inner ear are nearest neighbors. They share common sensory transduction elements called hair cells and are in

\section{KARGER}

E-Mail karger@karger.com www.karger.com/aud (c) 2020 The Author(s)

Published by S. Karger AG, Basel

Karger

Open access

This article is licensed under the Creative Commons AttributionNonCommercial-NoDerivatives 4.0 International License (CC BYNC-ND) (http://www.karger.com/Services/OpenAccessLicense). Usage and distribution for commercial purposes as well as any distribution of modified material requires written permission.
James O. Phillips, $\mathrm{PhD}$

Virginia Merrill Bloedel Hearing Research Center CD 176L, CHDD Building, Box 357923

University of Washington, Seattle, WA 98195 (USA)

E-Mail jop@uw.edu 
similar fluid compartments. The afferent fibers from these end organs are adjacent to each other in the internal auditory canal. As a result, both systems are often impacted by the same pathologies producing a combined loss of function. For many years, sensorineural hearing loss has been successfully treated with prosthetic devices designed to stimulate cochlear afferents electrically in response to sound stimulation. Such cochlear implants bypass the natural transduction elements in the inner ear which, when lost or injured, are often the cause of hearing loss. Similar devices have been developed to restore vestibular function. These devices use electrical stimulation of vestibular afferents, primarily the semicircular canal afferents, in response to motion stimulation, bypassing the hair cells of the ampullae of the semicircular canals [Golub et al., 2014; Guinand et al., 2015, Boutros et al., 2018].

Since both systems utilize common technologies and stimulation strategies, it should be possible to develop a single device that stimulates both the vestibular and cochlear afferents in response to both sound and motion, driving each system with its appropriate sensory input, and restoring function to both sensory systems.

We have recently developed and tested a vestibular implant in rhesus monkeys and ultimately in 4 human subjects. This device was studied longitudinally and was shown to produce vestibulo-ocular responses, electrically elicited compound action potentials, postural sway and motion percepts largely consistent with the responses predicted from activation primarily of the afferents of a single implanted semicircular canal [Phillips et al., 2013, 2015a].

Furthermore, such stimulation, at pulse frequencies and current levels sufficient to produce vestibular stimulation, did not produce sound sensation, pain, or visually observed or subjectively reported stimulation of the facial nerve. As a result of these initial experiments, a second device, with both cochlear and semicircular canal arrays, was constructed. Following the same strategy, the redesigned device in reduced form was implanted in rhesus monkeys, and then the full device was implanted in 3 human subjects with combined vestibular and severe-to-profound sensorineural hearing loss. The results of the initial safety and functionality studies have been published elsewhere [Rubinstein et al., 2019]. However, one important conceptual and practical feature of the stimulation was not fully evaluated in these studies.

In order for a combined vestibular and cochlear prosthesis to function optimally, it must be possible to stimu- late each sensory modality independently. This means that stimulation of the vestibular end organ does not elicit sound sensation, and stimulation of the cochlear end organ does not elicit motion sensation. However, it also means that combined stimulation of both end organs simultaneously should not produce interactions between the modalities such that sounds elicited by the cochlear arrays are altered by motion inputs from the vestibular arrays, and vice versa. Many years of studies on cochlear implants suggest that interactions between stimulation from different electrode sites are common due primarily to current spread. This is a primary limitation of current cochlear implant technology. Similarly, studies on vestibular prostheses suggest that interactions also exist with stimulation of individual canals eliciting eye movements suggesting current spread to adjacent canals or otolith organs. The primary question of this paper is whether such interactions also exist between sensory modalities during stimulation with a combined vestibular and cochlear implant.

\section{Methods}

This study was conducted in accordance with the Declarations of Helsinki and the Federal Policy for the Protection of Human Subjects: Notes and Rules. The research protocol was approved by the Human Subjects Division of the University of Washington. The US Food and Drug Administration approved this study under IDE G100075/R004. Written consent was obtained from each subject after a full discussion of the study, its risks, and its potential benefits.

Device

The device consisted of a Nucleus Freedom cochlear implant (Cochlear Ltd.) with 3 semicircular canal arrays, one Hybrid-L cochlear array, and a remote ground. The surface area of each of 2 stimulation sites on the semicircular canal array was $0.236 \mathrm{~mm}^{2}$ and the length of the array $2.5 \mathrm{~mm}$ (Fig. 1, sites E1-E6). The design allowed bipolar stimulation between sites on the array, but the studies described here utilized monopolar stimulation between the most distal site on each semicircular canal array (E2, E4, and E6) and the receiver stimulator case and a remote ground. The cochlear array consisted of a 16-mm Cochlear Hybrid-L intracochlear array, with 16 stimulation sites (Fig. 1, sites E7-E22). The Hybrid-L has been shown to provide very good speech discrimination with electrical stimulation only [Roland et al., 2016, 2018].

\section{Subjects}

Full details of the etiology and clinical evaluation of the patients was published previously [Rubinstein et al., 2019]. Briefly here, subject VI05 was implanted in the left ear. This subject had severeto-profound idiopathic sudden sensorineural hearing loss in the left ear with vertigo approximately 10 years prior to implantation 
Fig. 1. Cochlear vestibular neuroprosthesis. Electrode configurations are listed next to the appropriate leads. Each of the three canal electrodes has two linearly arranged stimulation sites, both intended to lie in the perilymphatic space adjacent to the ampulla of a single canal. The cochlear electrode has 16 stimulation sites. The most distal electrode (tip of the array) is the higher number electrode on each array. GND, ground.

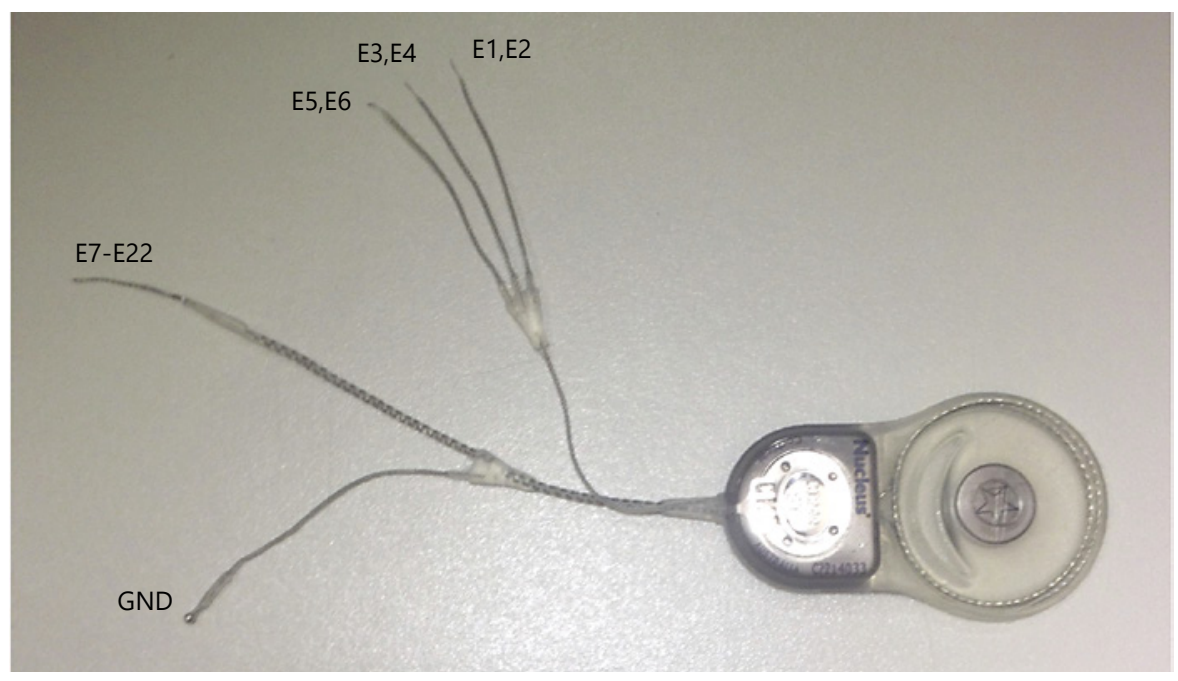

with the device. Subsequently, she had a complaint of chronic ataxia and oscillopsia due to bilateral vestibular areflexia. Subject VI06 was implanted in the left ear. She had long-standing bilateral Ménière's disease that had progressed to bilateral vestibular hypofunction with chronic ataxia and oscillopsia as well as sensorineural hearing loss. Subject VI07 was implanted in the right ear. $\mathrm{He}$ also had long-standing bilateral Ménière's disease and had previously received a left vestibular nerve section which produced profound deafness. He had been previously implanted with an Advanced Bionics $90 \mathrm{~K}$ cochlear implant in the left ear. He had severeto-profound sensorineural hearing loss in the right ear, ataxia, and oscillopsia.

\section{Surgery}

The vestibular and cochlear receiver stimulator was implanted under general anesthesia using a soft surgical technique [Golub et al., 2014; Phillips et al., 2015a]. Following a postauricular incision and mastoidectomy, the semicircular canals were blue lined, and small fenestrations were placed in the bony labyrinth adjacent to the ampullae. The tip of each semicircular canal lead was placed in the perilymphatic space, and the fenestrations were closed with fascia. The leads external to the fenestrations were tied down with suture. The Hybrid-L electrode array was implanted via the round window membrane with a fascia seal. No complications were observed during the surgical procedures. The subjects were monitored overnight in the hospital.

\section{Functional Assessment}

After 1 or 2 weeks of postsurgical recovery, the implanted receiver stimulator was activated in the laboratory. Laboratory testing was repeated at regular intervals. At each laboratory testing session, a standard clinical interface (Cochlear Programming Pod, Cochlear) attached to a laptop computer was used to record impedance measurements and vestibular electrically evoked compound action potentials for each stimulating electrode [Phillips et al., 2015a]. Bilateral recording of slow-phase eye movements during nystagmus elicited by short trains of biphasic pulse electrical stimulation was used to assess the efficacy of vestibular electrical stimulation.

\section{Electrical Stimulation}

The stimulation trains consisted of constant pulse rate, constant current amplitude biphasic pulses, $100 \mu$ s per phase with an 8 - $\mu$ s interphase gap, delivered between a stimulating electrode and both the case of the receiver stimulator and remote ground. Stimulation was controlled via a NIC-2 research interface (Cochlear) attached via a control pod and USB interface to a desktop computer running custom research software. All implanted electrodes were evaluated using such stimuli, with the current levels adjusted so that they did not exceed conservative safe charge limits for each electrode $(\leq 800 \mu \mathrm{A}$ at $100 \mu \mathrm{s} /$ phase for all stimulation sites; safety factor of 2 for safe charge limits for this electrode geometry). These stimulation parameters were chosen for test stimuli because they elicited consistent well-formed nystagmus with sustained slowphase eye velocity in both humans and rhesus monkeys, based on recording of electrically elicited eye movements with our first-generation devices in humans and monkeys, and our second-generation devices in monkeys [Phillips et al., 2015a, b]. The brief intermittent nature of the stimulation was designed to ensure subject safety and limit adaptation to the stimulation with repeated trials.

Stimulation of the cochlear array was conducted in a manner analogous to the stimulation of the vestibular electrodes. Brief 2-s trains of constant pulse frequency and constant current amplitude biphasic pulse stimulation were delivered to one of the stimulation sites on the cochlear electrode. Each site was mapped to determine the range of stimulation that produced clearly perceptible sound sensations without being aversively loud. Typically, current levels used during testing were assessed to be approximately halfway between threshold for a sound percept and the levels that were reported to be aversive (rating of 4-6 on a scale of 1-10). The electrical stimulation typically has a pulse frequency of 300 pps, with 100 $\mu$ ser phase and an 8 - $\mu$ s interphase gap so that it was comparable to the stimulation delivered to the vestibular electrodes. The longer phase duration but lower current amplitude stimulation was also chosen to reduce current spread to the adjacent end organs.

\section{Combined (Interleaved) Stimulation}

To assess the interaction between stimuli delivered to the cochlear and vestibular arrays, combined stimulation at two sites was 
Fig. 2. Representative stimulation currents series from the most distal site on the lateral and anterior canal electrode arrays. a Horizontal slow-phase velocities of nystagmus elicited by stimulation of the most distal site on each electrode array at different current levels. Up is rightward velocity, and down is leftward velocity. b Vertical slow-phase velocities of nystagmus elicited by stimulation of the most distal site on each electrode array at different current levels. Up is upward velocity, and down is downward velocity. The stimulation parameters were 2 -s train at 300 pps with $100 \mu \mathrm{s} /$ phase and an 8 - $\mu$ s gap. Multiple trials of stimulation are represented. Symbols are means, and error bars are 1 SD. Colored symbols indicate the stimulated canal array.

\section{Current series VI05}
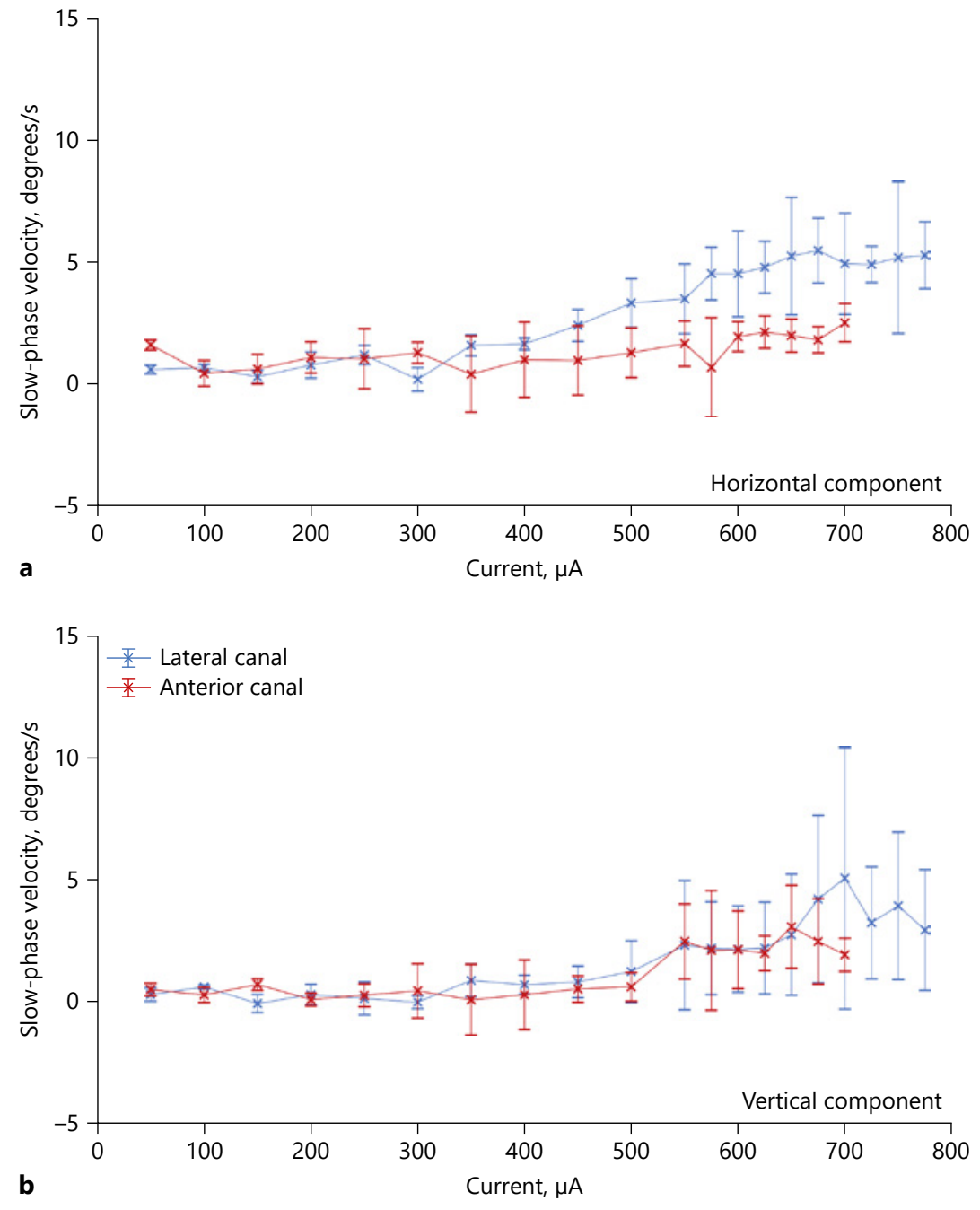

performed. Such stimulation consists of interleaved pulses delivered sequentially across the various arrays, resulting in sequentially delivered pulses in each array in close temporal proximity (5 $\mu s)$. These interleaved stimulation trials were presented with short 2-s trains of constant current and constant pulse frequency, and could be compared directly to the single-site stimulation.

\section{Binocular Eye-Movement Recording and Data Analysis}

Eye movements resulting from stimulation were recorded with the subjects seated in a research rotator (Micromedical, Chatham, IL, USA). The subjects wore a chest and lap belt during testing, and the head rested against a headrest. The eye movements were recorded in darkness with a head-mounted binocular IR VOG system sampling in 2D at $100 \mathrm{~Hz}$ (Neuro Kinetics). In parallel with the eye movement recordings, stimulation and data sync pulses were digitized online at $1 \mathrm{kHz}$ using Spike2 software running on a CED Power 1401 computer interface (Cambridge Electronic De- sign, Cambridge, UK). The stimulation software also kept a record of all stimulation pulse parameters for each stimulus trial. Data analysis was performed offline. Eye movement data and stimulus files were exported and aligned temporally. The velocities of the slow phases of evoked nystagmus were obtained by differentiation of the eye position data following removal of the fast phases using a velocity criterion. The velocity of individual slow phases was determined by a least-squares fit to the data, and average slow-phase velocity resulting from a single stimulus train was calculated as the time-weighted average of all slow phases occurring during a 2-s interval beginning $10 \mathrm{~ms}$ after the onset of electrical stimulation. The resulting slow-phase velocities were plotted as a current series at a fixed pulse frequency similar to the data for the lateral and anterior canal stimulation for subject VI05 shown in Figure 2. This subject had a relatively weak response to electrical stimulation across current amplitude for both canals, while other subjects had higher velocity responses. 
Normalized pitch difference

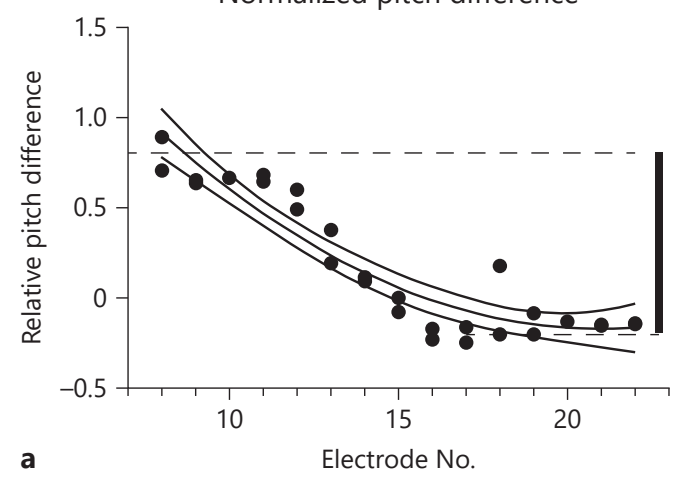

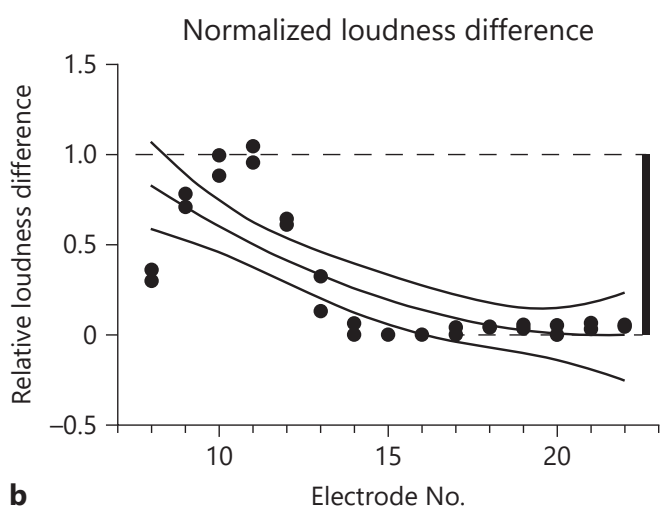

Fig. 3. Calculation of normalized loudness and pitch differences. Likert scale judgments were obtained using a standard cochlear electrode in the center of the array as the reference. Stimuli were delivered for $2 \mathrm{~s}$ at $300 \mathrm{pps}$ at a fixed current level that was adjusted to produce a comfortable sound level with stimulation of the reference electrode (E15). Immediately after the presentation of the reference stimulation, a second stimulus was delivered from another electrode in the cochlear array whose electrode number is listed on the $x$ axis. Two or more trials were performed for each electrode combination. Each trial represents a single dot. The dif- ference between the largest positive difference (average for a given array combination) and the largest negative difference (average for a given array combination) was then calculated from the data across the array (thick vertical bar). This was arbitrarily assigned a value of 1 . All subsequent judgments were normalized to this value. Both graphs plot individual perceptual judgments fit by a second-order polynomial and bounded by the $95 \%$ confidence interval of the fit. a Normalized pitch differences across the cochlear array relative to electrode \#15. b Normalized loudness differences across the cochlear array relative to electrode \#15.
Likert Scale Assessment of Perceived Pitch and Loudness

In order to assess differences in the pitch and loudness of the sensations evoked by the electrical stimulation of two individual cochlear stimulation sites, or a single cochlear site with and without concurrent vestibular electrical stimulation, we used a simple Likert scale. Immediately after presentation of two cochlear electrical stimulation trains, subjects were instructed to mark a linear scale indicating the pitch difference between the two stimuli. The center of the scale indicated no difference, while the right of the scale indicated that the second stimulus was higher pitch that the first, and the left of the scale indicated that the pitch was lower. The distance from the center estimated the magnitude of the difference. A similar scale was used for estimation of loudness difference. It should be noted that such estimates do not accurately assess pure pitch or loudness percepts per se, but rather it is thought that combinations of pitch, timbre, and loudness contribute to such judgments in complex combinations. In the following paragraphs we are referring to nominal pitch and loudness as assessed with this specific method.

Comparisons of Pitch and Loudness Differences for Sites across the Cochlear Array

In order to calibrate the Likert scale assessments of interactions between vestibular and cochlear site stimulation, we first assessed the differences in pitch and loudness across the cochlear array. It is known that significant differences exist in pitch and loudness between different sites on a cochlear array, and monitoring such levels at each site is an essential step in mapping such a device to produce optimal functional stimulation. To assess differences between electrodes in the cochlea, we chose a single central site to provide reference stimulation (typically E15 or E16). This site was half way down the cochlear array. We then compared stimulation at this site with a second stimulation (with identical stimulation parameters) at a second site on the array. The resulting difference in pitch and loudness were recorded by subjects on the Likert scale. An example of data from such procedures is presented in Figure 3 for subject VI06. The reference site was site E15.

As can be seen in Figure 3a, differences in pitch varied across the electrode, with the pitch being higher at more proximal sites (closer to the base of the cochlea) and lower at more distal sites (closer to the apex of the cochlea). Repetition of the stimulation produced comparable pitch difference percepts. Similarly, Figure $3 \mathrm{~b}$ shows difference in loudness for the same electrode comparisons. In order to calibrate our cross-modal interaction comparisons, with combined vestibular and cochlear stimulation, we normalized all of our results based on data such as that in Figure 3 for each subject. We arbitrarily assigned a difference value of 1.0 for the greatest difference of pitch and loudness between sites across the entire cochlear array. This was calculated by taking the highest and lowest average value for each electrode site in our comparisons, and summing the absolute differences, producing a single magnitude value for nominal pitch and a single value for loudness (Fig. 3, thick vertical bars). That value was normalized to 1.0 for all subsequent comparisons. 
Fig. 4. Effect of interleaved stimulation on the slow-phase velocity of nystagmus elicited by stimulation of the most distal electrode in the lateral canal of subject VI07. a Horizontal slow-phase velocities of nystagmus elicited by stimulation of the most distal site on the electrode array at different current levels. Up is rightward velocity, and down is leftward velocity. b Vertical slowphase velocities of nystagmus elicited by stimulation of the most distal site on each electrode array at different current levels. Up is upward velocity, and down is downward velocity. The stimulation parameters were 2 -s train at 300 pps with $100 \mu$ s/phase and an $8-\mu$ s gap. Multiple trials of stimulation are represented. Symbols are means, and error bars are $1 \mathrm{SD}$. Colored symbols indicate the canal and cochlear stimulation site combinations stimulated. ${ }^{*} p<0.05$ : statistically significant result.
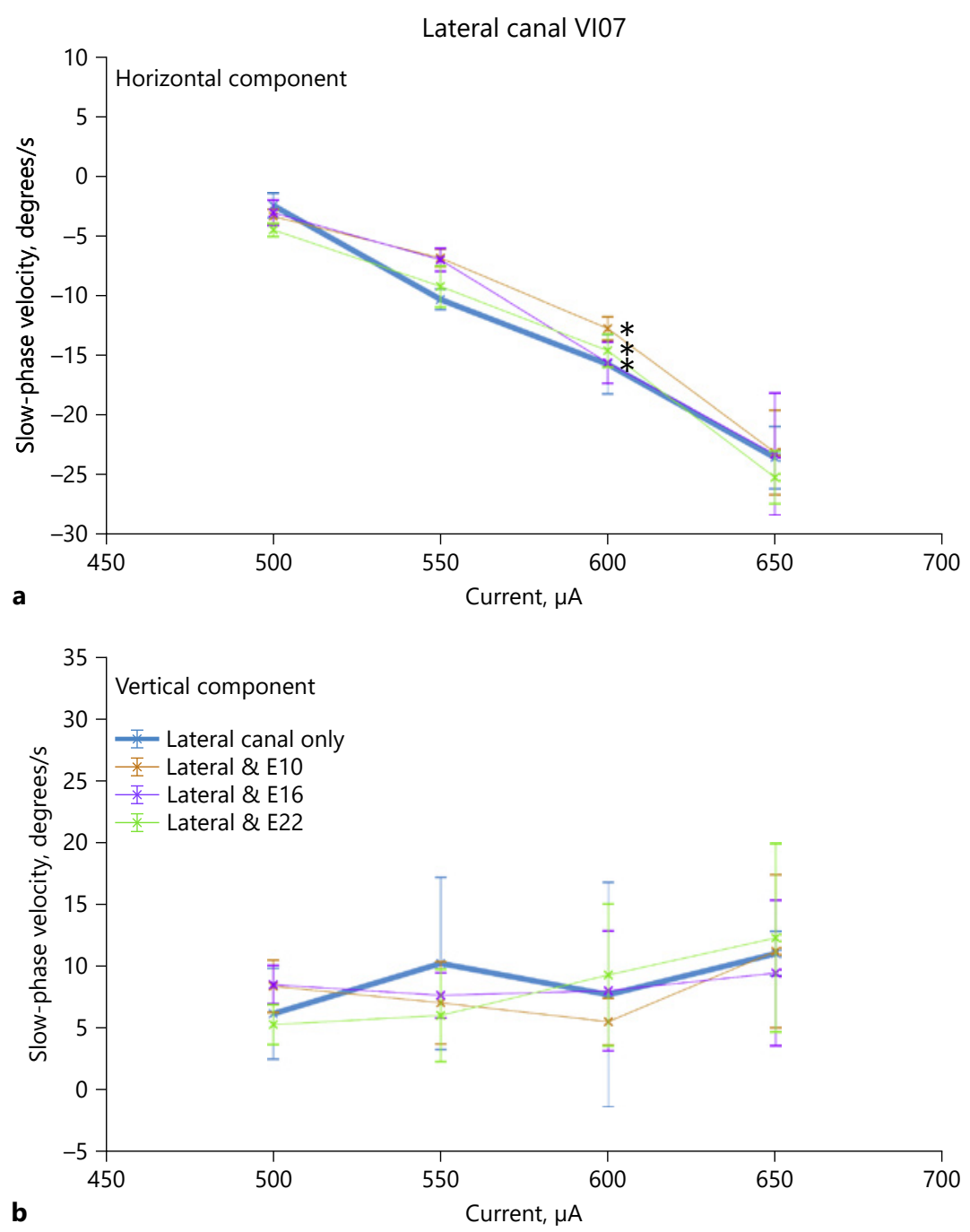

\section{Results}

All subjects responded to 2-s trains of biphasic pulse electrical stimulation of the canal electrodes with slowphase eye movements and nystagmus. Importantly, no subject experienced any sensation of sound during canal electrode stimulation. The elicited sensations were of movement only. This suggested that stimulation of the vestibular modality alone was effective and independent of effective stimulation of other sensory modalities. In subject VI05, the slow-phase velocities were very low across all stimulus currents (Fig. 2), but in the other two subjects the eye movements were largely in the plane of the canal and the velocities, which scaled with current were higher. For example, in Figure 4, the thick line indicates the slow-phase velocities elicited by right canal only stimulation in subject VI07. At the highest current amplitudes, the leftward horizontal eye velocities elicited by such stimulation were 24 degrees/s (Fig. 4a). There was also an upward directed vertical velocity at the highest currents as well (11 degrees/s), which may have been due to current spread to the anterior canal or the utricle (Fig. 4b).

Stimulation of cochlear electrodes similarly produced sound sensations in all of our subjects. Importantly, stimulation of the cochlear electrodes alone did not produce sensation of movement, slow-phase eye velocity, or nystagmus. This demonstrates that stimulation of the cochle- 


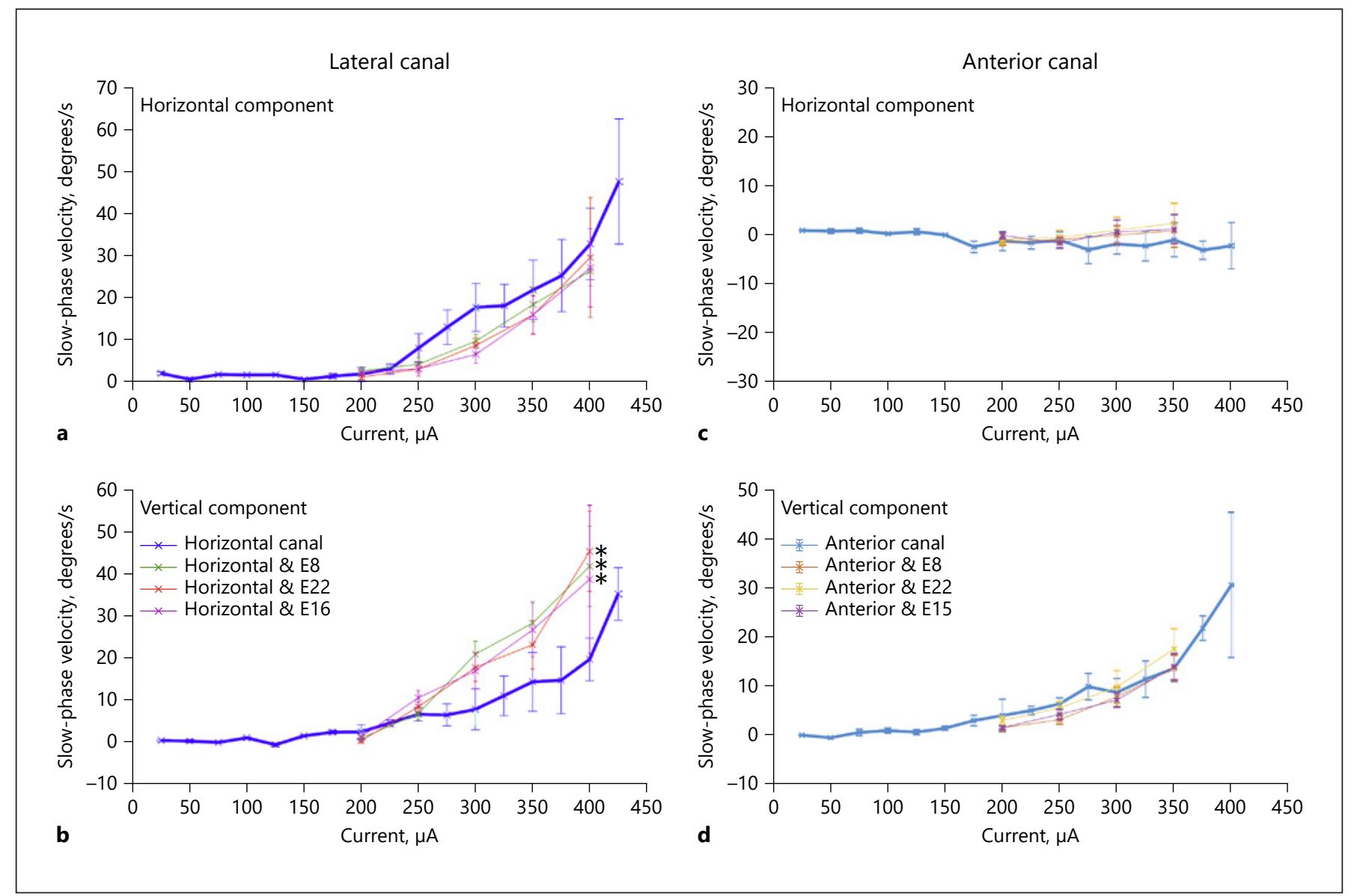

Fig. 5. Effect of interleaved stimulation on the slow phase velocity of nystagmus elicited by stimulation of the most distal electrode in the array in each of two separate canals of subject VI06. a Horizontal slow-phase velocities of nystagmus elicited by stimulation of the most distal site on the lateral canal electrode array at different current levels. b Vertical slow-phase velocities of nystagmus elicited by stimulation of the most distal site on the lateral canal electrode array at different current levels. c Horizontal slow-phase velocities of nystagmus elicited by stimulation of the most distal site on the anterior canal electrode array at different current levels. $\mathbf{d}$ Vertical

ar electrodes was effective in driving the auditory modality without driving the vestibular sensory modality. This independence is an important feature of a combined prosthesis.

Our experiments were constructed to evaluate interactions during the combined stimulation that would occur under normal operating conditions of the device when it was supplementing both sensory modalities simultaneously. To do this, we interleaved 2-s stimulation of the semicircular canals with stimulation of the cochlear array at a current level which produced a comfortable and clear

slow-phase velocities of nystagmus elicited by stimulation of the most distal site on the anterior canal electrode array at different current levels. For horizontal velocities, up is rightward velocity, and down is leftward velocity. For vertical velocities, up is upward velocity, and down is downward velocity. The stimulation parameters were 2 -s train at 300 pps with $100 \mu$ s/phase and an 8 - $\mu$ s gap. Multiple trials of stimulation are represented. Symbols are means, and error bars are $1 \mathrm{SD}$. Colored symbols indicate the canal and cochlear stimulation site combinations stimulated. ${ }^{*} p<0.05$ : statistically significant result.

percept of sound during stimulation of a central site in the array (either E15 or E16). The primary research question was whether such combined stimulation altered the response to stimulation of a single site alone.

\section{Combined Stimulation Effects on Vestibular Inputs}

Initially, we evaluated the effect of cochlear stimulation on slow-phase eye velocity. Subject VI05 had eye velocities which were minimal, and so this evaluation was not performed in that subject. As shown in Figure 4, subject VI07 had higher eye velocities largely in the plane of 
Pitch and loudness interactions VI06

Lateral canal

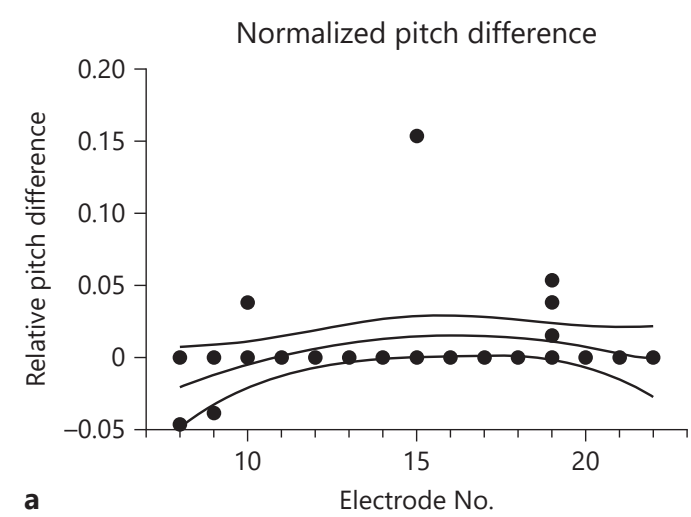

a

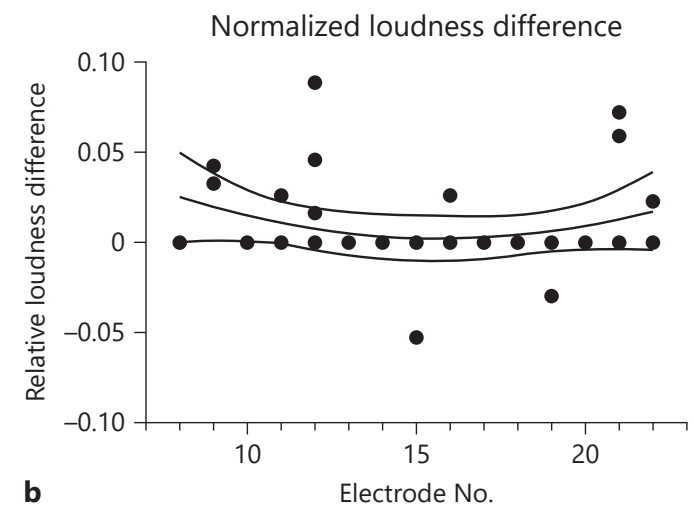

Fig. 6. Pitch and loudness interactions comparing stimulation of a cochlear array site alone with a combination of stimulation of that site with the interleaved stimulation of the distalmost site on the lateral canal array at threshold for perceived motion in subject VI06. Both graphs plot individual perceptual judgments fit by a second-order polynomial and bounded by the $95 \%$ confidence interval of the fit. a Normalized perceived pitch differences between cochlear stimulation alone and cochlear stimulation with interleaved vestibular array stimulation. Positive values indicate an increase in perceived pitch with combined stimulation, and negative values indicate a decrease. b Normalized perceived loudness differences between cochlear stimulation alone and cochlear stimulation with interleaved vestibular array stimulation. Positive values indicate an increase in perceived loudness with combined stimulation, and negative values indicate a decrease. Electrode number on the cochlear array is indicated by the values on the abscissa of each graph. A value of 1.0 on the ordinate of either graph corresponds to the maximum difference for a given measure between any two electrodes on the cochlear array for subject VI06. the stimulated canal, so this test could be performed meaningfully. Figure 4 shows that, in fact, effective stimulation of the cochlear electrodes in subject VI07, which elicited clear sound sensations, did produce small but significant changes in eye velocity in the horizontal (Fig. 4a) but not the vertical (Fig. 4b) dimension. Stimulation was performed across a full range of current levels in the right lateral canal of this subject, with concurrent stimulation of the basal, middle, and apical electrodes of the cochlear array (E10, E16, and E22). The concurrent stimulation vertical velocity curves (Fig. 4b) consistently overlap the single canal velocity curves, but due to the low variability of the horizontal slow phase velocities, there are small but significant differences between the velocities resulting from lateral canal stimulation alone and the velocities resulting from stimulation of all electrodes. We performed an ANOVA comparing lateral canal horizontal velocities with those of the combinations of E10, E16, and E22 with the lateral canal, and all were found to be significantly different $(p<0.05)$. Vertical velocities were not significantly different. Such small differences, while statistically significant, are probably of little clinical significance.
Similar experiments were performed for the anterior and lateral canals of subject VI06, combined with stimulation of the basal, middle, and apical electrodes of the cochlear array (E8, E15 or E16, and E22). The results of these experiments are shown in Figure 5. For the anterior canal electrodes, no significant changes were seen between stimulation of the canal alone and combined stimulation with the cochlear electrodes. Both horizontal and vertical components showed overlapping velocities in the canal alone and combined stimulation conditions (Fig. 5c, d). However, for the lateral canal, a large and potentially clinically significant difference was seen at higher lateral canal stimulation current levels between lateral canal stimulation alone and stimulation combined with stimulation of each of the cochlear electrodes. While there were small but significant differences seen in the horizontal component of the evoked slow-phase eye velocities (Fig. 5a), there were large shifts toward higher vertical velocities with combined stimulation (Fig. 5b). We performed an ANOVA comparing lateral canal horizontal and vertical velocities with those of the combinations of E8, E16, and E22 with the lateral canal, and all 
Pitch and loudness interactions VI07 Lateral canal

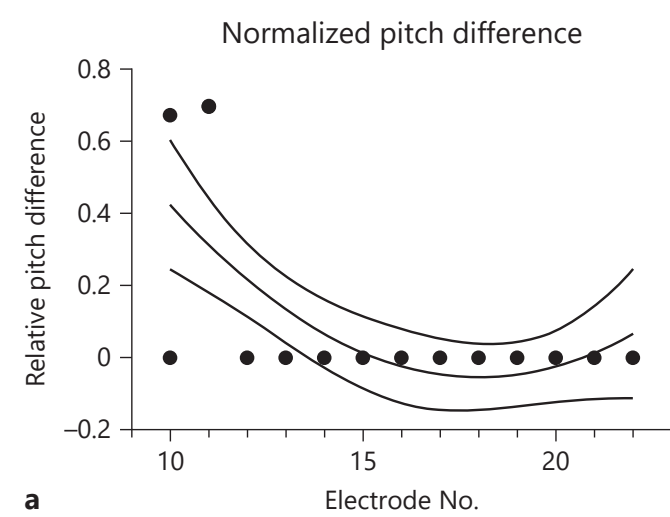

a

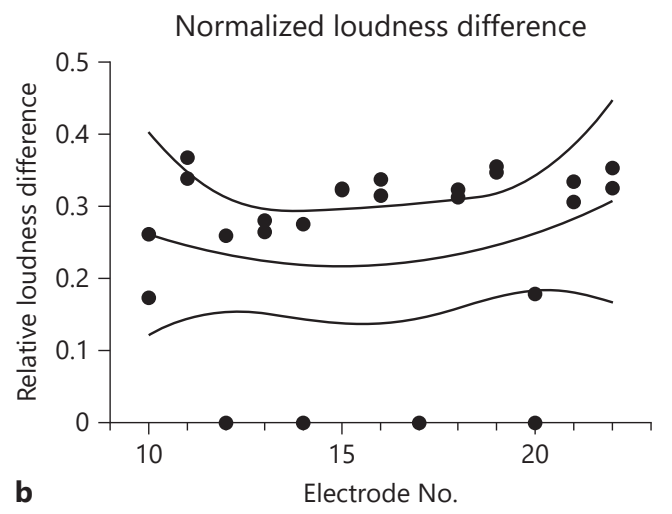

Fig. 7. Pitch and loudness interactions comparing stimulation of a cochlear array site alone with a combination of stimulation of that site with the interleaved stimulation of the distalmost site on lateral canal array at threshold for perceived motion in VI07. Both graphs plot individual perceptual judgments fit by a second-order polynomial and bounded by the $95 \%$ confidence interval of the fit. a Normalized perceived pitch differences between cochlear stimulation alone and cochlear stimulation with interleaved vestibular array stimulation. Positive values indicate an increase in perceived pitch with combined stimulation, and negative values indicate a decrease. b Normalized perceived loudness differences between cochlear stimulation alone and cochlear stimulation with interleaved vestibular array stimulation. Positive values indicate an increase in perceived loudness with combined stimulation, and negative values indicate a decrease. Electrode number on the cochlear array is indicated by the values on the abscissa of each graph. A value of 1.0 on the ordinate of either graph corresponds to the maximum difference for a given measure between any two electrodes on the cochlear array for subject VI07. were found to be significantly different $(p<0.05)$. At some currents, the vertical slow-phase velocities of the evoked movements more than doubled during combined stimulation. This means that both the magnitude and the direction of the eye movements, and presumably the vestibular input that this reflects, were significantly altered.

\section{Combined Stimulation Effects on Auditory Inputs}

We investigated the effects of combined vestibular stimulation on the resulting estimation of nominal pitch and loudness during stimulation at sites across the cochlear array in all 3 subjects. The experiments were performed at canal current levels at threshold for evoking a sensation of motion, typically comparable to the threshold for evoking low velocity eye movements. Current levels for the cochlear electrodes were held constant across the array, at levels that evoked clear and comfortable sensations of sound for sites in the middle of the array.

Results of combined stimulation experiments for subject VI06 are seen in Figure 6, which plots the levels of perceived pitch differences and perceived loudness between cochlear site stimulation alone and combined lateral canal and cochlear site stimulation. In this and all comparable experiments, stimulation of each electrode combination was performed twice, resulting in at least 2 points per cochlear electrode number. The data are normalized so that the estimation differences are relative to the greatest differences observed between two sites on the cochlear array using the same estimation technique. As can be seen in Figure 6a, there was very little difference in pitch or loudness between cochlear site stimulation and combined stimulation for this subject. Those changes that were reported tended to indicate an increase in pitch and loudness with combined stimulation.

Comparable experiments were also performed in subject VI07, combining lateral canal stimulation with stimulation of sites along the cochlear array. The results here were quite different (Fig. 7). This subject reported an increase in pitch, but only for the most basal electrodes, which were nearest to the lateral canal (Fig. 7a). The increases tended to be large, ranging from an average of $30-70 \%$ of the total differences seen across the sites of the 


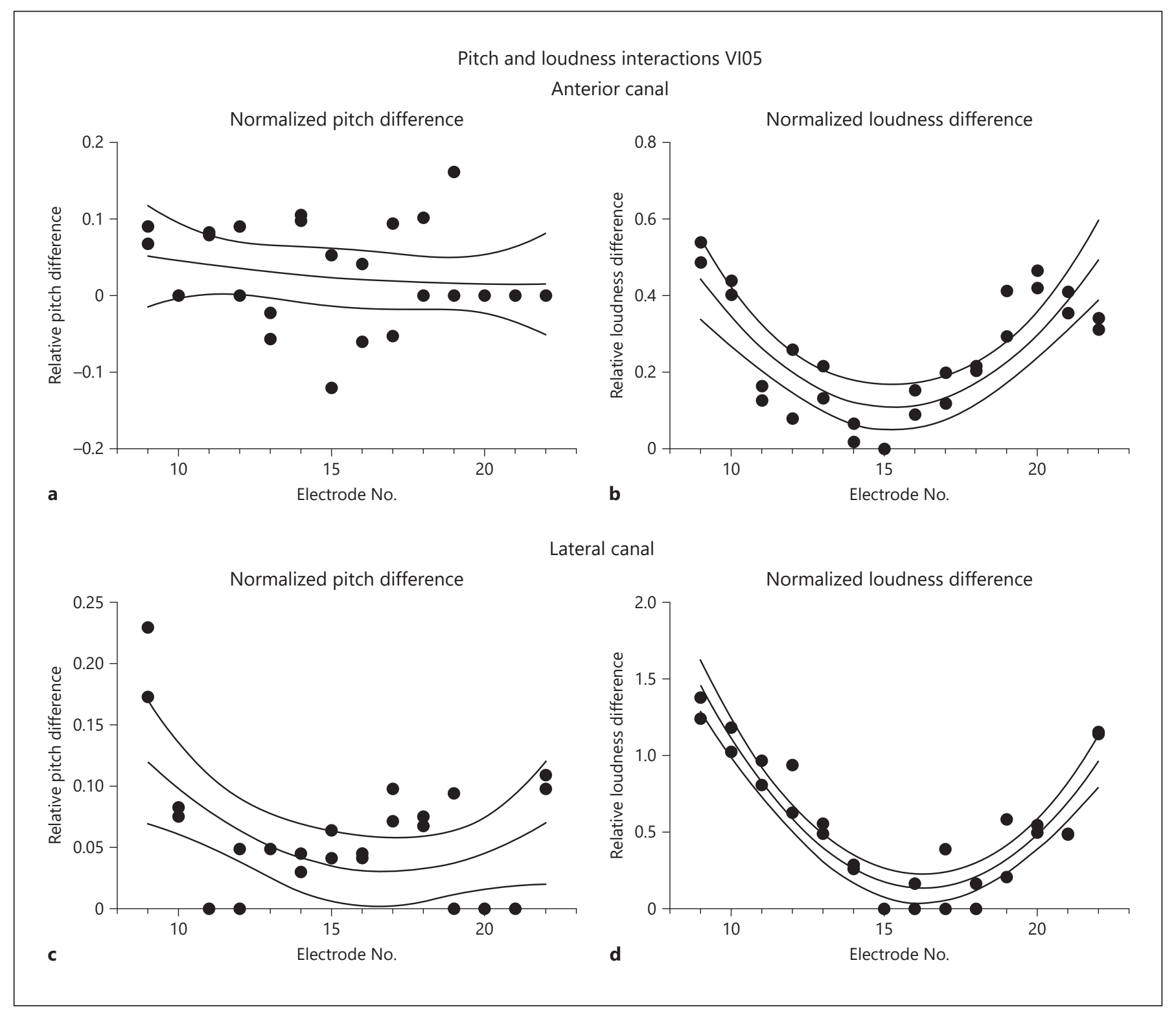

Fig. 8. Pitch and loudness interactions comparing stimulation of a cochlear array site alone with a combination of stimulation of that site with the interleaved stimulation of the distalmost site on the anterior or lateral canal array at threshold for perceived motion in VI05. All graphs plot individual perceptual judgments fit by a second-order polynomial and bounded by the $95 \%$ confidence interval of the fit. a Normalized perceived pitch differences between cochlear stimulation alone and cochlear stimulation with interleaved vestibular anterior canal array stimulation. b Normalized perceived loudness differences between cochlear stimulation alone and cochlear stimulation with interleaved vestibular anterior canal array stimulation. c Normalized perceived pitch differences be-

tween cochlear stimulation alone and cochlear stimulation with interleaved vestibular lateral canal array stimulation. d Normalized perceived loudness differences between cochlear stimulation alone and cochlear stimulation with interleaved vestibular lateral canal array stimulation. Positive values indicate an increase in perceived pitch or loudness with combined stimulation, and negative values indicate a decrease. Electrode number on the cochlear array is indicated by the values on the abscissa of each graph. A value of 1.0 on the ordinate of either graph corresponds to the maximum difference for a given measure between any two electrodes on the cochlear array for subject VI05. 
cochlear array alone. There were no pitch interactions for sites further distal (middle and apical sites) on the array. For loudness estimation, the differences were large and relatively consistent, with increases in loudness averaging approximately $30 \%$ for combined stimulation with sites across the cochlear array.

Finally, the same experiments were performed for stimulation of both the anterior and lateral canals of subject VI05. The results of these experiments are displayed in Figure 8. For the anterior canal, the differences in pitch between canal alone and combined canal and cochlear stimulation were relatively small (Fig. 8a). On average, these differences were less than $10 \%$ of the range of differences across the cochlear array for cochlear stimulation alone. However, the differences in loudness estimation were quite large and showed a topographic organization across the cochlear array, such the basal and apical electrodes showed the greatest increases in loudness with combined stimulation (on average approx. 42 and $46 \%$ of the largest differences between sites on the cochlear array for the basal and apical electrodes, respectively).

Even more importantly, the addition of anterior canal stimulation produced large changes in the estimated loudness of stimulation of individual cochlear sites (Fig. 8b). These changes were highest for the most basal and most apical electrodes, and seemed to vary systematically between them. The magnitude of the difference was between 55 and $0 \%$ across the array, with no change in loudness at the middle of the array (electrode E15).

Comparable measures were also obtained for addition of lateral canal stimulation to the stimulation of the cochlear sites in subject VI05. A similar effect was observed for the lateral canal combinations. For the addition of stimulation from this canal, all perceived pitch changes were increases in pitch or no change. The magnitude of the pitch changes was greatest with stimulation of sites at the basal end of the cochlear array, which showed an average change of $20 \%$ for electrode E9. Loudness changes showed the same pattern for the lateral canal stimulation combinations that was observed for the anterior canal stimulation combinations. Combined stimulation always produced an increase in loudness, with the greatest increases occurring for stimulation sites at the basal and apical ends of the cochlear array. In this case, however, the loudness increases exceeded the maximum difference in loudness observed across all of the electrodes in the cochlear array. The magnitude of the observed increase in loudness was at most about 1.3 times the difference observed between sites across the cochlear array.

\section{Discussion}

Taken together, these results suggest that both statistically and potentially clinically significant changes in nominal pitch and loudness can occur when stimulation with a vestibular array is combined with stimulation with a cochlear array. Likewise, these data show that stimulation with a cochlear array can alter the vestibular signals, in direction and magnitude, elicited by stimulation with the vestibular arrays. These changes in velocity are in some cases very large, exceeding 20 degrees/s. Such changes greatly exceed the velocities of clinically significant spontaneous and positional nystagmus, and the velocities elicited by a clinically significant Tullio phenomenon secondary to superior semicircular canal dehiscence [Cremer et al., 2000]. Such changes occur despite the fact that stimulation of either the cochlear sites or the canal sites alone produce no cross-modal sensations; i.e., canal sites do not produce sound percepts, and cochlear sites do not produce motion percepts or slow-phase eye velocity when activated alone. With combined stimulation, such changes are idiosyncratic from subject to subject, and do not necessarily occur together. For example, in subject VI06, large changes were observed in the slow-phase velocity of the elicited nystagmus in response to lateral canal stimulation at higher current levels, but no changes were elicited in the auditory percept of pitch or loudness in response to cochlear stimulation when threshold stimulation of the lateral canal was combined.

The observed auditory effects seem to be occurring primarily at the ends of the cochlear array. For the basal stimulation this makes sense if the underlying mechanism is current spread or changes in current path within the inner ear, since these sites are located at the shortest distance from the vestibular end organs. The effects at the apical electrodes are almost as great in some subjects. The reason for this is unclear. It is tempting to speculate that it is because the electrode array has advanced around the cochlear spiral to again approach the vestibular end organs. However, this is unlikely since the cochlea is surrounded by higher impedance bone at this point, and the facial nerve is far closer than the vestibular labyrinth, yet we observed no low threshold facial stimulation during our interaction studies. Since we did not objectively assess facial nerve stimulation with electromyography [e.g., Cushing et al., 2006, 2009], we cannot rule out the possibility that subliminal stimulation did occur.

Another second possible, although less plausible mechanism for the interactions that we observe might be 
related to cross-modal interaction in the natural processing of vestibular and auditory signals in the central nervous system. Indeed, some experiments suggest that orientation information can be successfully conveyed by prosthetic stimulation of the auditory system with electrical stimuli, and that vestibular responses can be driven by stimulation with cochlear implants [Eisenberg et al., 1982; Buchman et al., 2004; Cushing et al., 2008, Gnanasegaram et al, 2016; Parkes et al., 2017]. Regardless of the underlying mechanism, the data presented here suggest that these changes can be large relative to differences between sites within the cochlear array, and also large relative to the overall slow-phase eye velocities elicited during stimulation of the canals.

These experiments were predicated on the assumption that the optimal situation for construction of a combined vestibular and cochlear prosthesis would be to have total independence of the sensory modalities to which the electrode stimulations are contributing. Put simply, it seems suboptimal to have head motion drive changes in sound quality, or to have auditory stimulation drive changes in the motion inputs to the brain. However, these interactions are not particularly surprising and may not limit the benefits derived from such a device. Similar experiments examining the combined stimulation of multiple canals have suggested that these inputs also fail to sum linearly when combined [Phillips et al., 2018]. Current spread between intended afferent targets and adjacent potential targets have always been a challenge to the development of prosthetic neural implants [Phillips et al., 2015a; Hedjoudje et al., 2019]. Hypothetically, the same strategies that have been used to sharpen the frequency tuning of cochlear implants can be used to reduce the interactions observed here. For example, multipolar stimulation can be used to steer current in cochlear implants [DeVries and Arenberg, 2018].

There are many things we still do not know based on the limited observations possible here as part of a larger safety and functionality study. For example, we do not know whether, in subjects with preserved hearing and a vestibular implant, natural sound quality is affected by vestibular stimulation. The experiments here were conducted with monopolar stimulation, and many of the interactions occurred at higher current levels. We do not know whether bipolar stimulation with an adjacent return can reduce or eliminate the effects shown here. We do not know whether other effects on vestibular sensory processing, such as postural sway or motion perception threshold are similarly affected by cochlear stimulation. We do not know whether parametric changes in stimula- tion direction, interphase gap or pulse frequency can change these interactions. Indeed, based on the limited experiments performed here, we do not even know the precise nature of the changes in the quality of sound that are evoked by combined stimulation. We simply know that there are changes that map to the nominal percepts of pitch and loudness in our subjects. Ultimately, these experiments reveal a limitation to the current strategies for driving a combined vestibular and cochlear implant. Future experiments will be critical to allow us to fully understand the phenomenon, and perhaps ultimately decrease or eliminate any such unwanted interactions in these devices.

\section{Acknowledgments}

The authors would like to acknowledge the contribution of Kaibao Nie in the development of the software interface used in these experiments.

\section{Statement of Ethics}

This study was conducted in accordance with the Declarations of Helsinki and the Federal Policy for the Protection of Human Subjects: Notes and Rules. The research protocol was approved by the Human Subjects Division of the University of Washington. The US Food and Drug Administration approved this study under IDE G100075/R004. Written consent was obtained from each subject after a full discussion of the study, its risks, and its potential benefits.

\section{Disclosure Statement}

The University of Washington and authors J.O. Phillips, L. Ling, and J.T. Rubinstein have intellectual property associated with the device used in these experiments. J.T. Rubinstein is a paid consultant for Cochlear Ltd.

\section{Funding Sources}

This work was supported by NIH grant R01-DC-014002.

\section{References}

Boutros P, Rahman M, Valentin N, Della Santina CC. First-in-human clinical trial of the MVI ${ }^{\mathrm{TM}}$ multichannel vestibular implant: continuous restoration of the human vestibulo-ocular reflex. ARO abstracts. San Diego: ARO; 2018.

Buchman CA, Joy J, Hodges A, Telischi FF, Balkany TJ. Vestibular effects of cochlear implantation. Laryngoscope. 2004 Oct; 114(10 Pt 2 Suppl 103):1-22. 
Cremer PD, Minor LB, Carey JP, Della Santina CC. Eye movements in patients with superior canal dehiscence syndrome align with the abnormal canal. Neurology. 2000 Dec;55(12):1833-41.

Cushing SL, Chia R, James AL, Papsin BC, Gordon KA. A test of static and dynamic balance function in children with cochlear implants: the vestibular olympics. Arch Otolaryngol Head Neck Surg. 2008 Jan;134(1):34-8.

Cushing SL, Papsin BC, Gordon KA. Incidence and characteristics of facial nerve stimulation in children with cochlear implants. Laryngoscope. 2006 Oct;116(10):1787-91.

Cushing SL, Papsin BC, Strantzas S, Gordon KA. Facial nerve electromyography: a useful tool in detecting nonauditory side effects of cochlear implantation. J Otolaryngol Head Neck Surg. 2009 Apr;38(2):157-65.

DeVries L, Arenberg JG. Current focusing to reduce channel interaction for distant electrodes in cochlear implant programs. Trends Hear. 2018 Jan-Dec;22:2331216518813811.

Eisenberg LS, Nelson JR, House WF. Effects of the single-electrode cochlear implant on the vestibular system of the profoundly deaf adult. Ann Otol Rhinol Laryngol Suppl. $1982 \mathrm{Mar}-$ Apr;91(2 Pt 3):47-54.

Gnanasegaram JJ, Parkes WJ, Cushing SL, McKnight CL, Papsin BC, Gordon KA. Stimulation from cochlear implant electrodes assists with recovery from asymmetric perceptual tilt: evidence from the subjective visual vertical test. Front Integr Neurosci. 2016;10:32.
Golub JS, Ling L, Nie K, Nowack A, Shepherd SJ, Bierer SM, et al. Prosthetic implantation of the human vestibular system. Otol Neurotol. 2014 Jan;35(1):136-47.

Guinand N, van de Berg R, Cavuscens S, Stokroos RJ, Ranieri M, Pelizzone M, et al. Vestibular implants: 8 years of experience with electrical stimulation of the vestibular nerve in $11 \mathrm{pa}-$ tients with bilateral vestibular loss. ORL J Otorhinolaryngol Relat Spec. 2015;77(4): $227-40$.

Hedjoudje A, Hayden R, Dai C, Ahn J, Rahman M, Risi F, et al. Virtual Rhesus Labyrinth Model Predicts Responses to Electrical Stimulation Delivered by a Vestibular Prosthesis. J Assoc Res Otolaryngol. 2019 Aug;20(4): 313-39.

Parkes WJ, Gnanasegaram JJ, Cushing SL, McKnight CL, Papsin BC, Gordon KA. Vestibular evoked myogenic potential testing as an objective measure of vestibular stimulation with cochlear implants. Laryngoscope. 2017 Feb;127(2):E75-81.

Phillips C, Defrancisci C, Ling L, Nie K, Nowack A, Phillips JO, et al. Postural responses to electrical stimulation of the vestibular end organs in human subjects. Exp Brain Res. 2013 Aug; 229(2):181-95.
Phillips C, Ling L, Oxford T, Nowack A, Nie K, Rubinstein JT, et al. Longitudinal performance of an implantable vestibular prosthesis. Hear Res. 2015a Apr;322:200-11.

Phillips JO, Ling L, Nie K, Jameyson E, Phillips CM, Nowack AL, et al. Vestibular implantation and longitudinal electrical stimulation of the semicircular canal afferents in human subjects. J Neurophysiol. 2015b Jun;113(10): 3866-92.

Phillips J, Ling L, Phillips C, Nowack A, Nie K, Rubinstein J. Combined stimulation of multiple canals to map a vestibular prosthesis. 30th Barany Society Abstracts. J Vestib Res. 2018;28(1-2):i-281 OP-06-6.

Roland JT Jr, Gantz BJ, Waltzman SB, Parkinson AJ. Long-term outcomes of cochlear implantation in patients with high-frequency hearing loss. Laryngoscope. 2018 Aug; 128(8): 1939-45.

Roland JT Jr, Gantz BJ, Waltzman SB, Parkinson AJ; Multicenter Clinical Trial Group. United States multicenter clinical trial of the cochlear nucleus hybrid implant system. Laryngoscope. 2016 Jan;126(1):175-81.

Rubinstein JT, Ling L, Nowack A, Nie K, Phillips JO. Results from a second-generation vestibular implant in human subjects: diagnosis may impact electrical sensitivity of vestibular afferents. Otol Neurotol. Forthcoming. 